\title{
Dynamic collimator optimization compared with fixed collimator angle in arc-based stereotactic radiotherapy: a dosimetric analysis
}

\author{
Christopher M. Lee, M.D., Gordon A. Watson, M.D., Ph.D., \\ and Dennis D. LeavitT, Ph.D.
}

Departments of Radiation Oncology, University of Utah and Huntsman Cancer Institute, and LatterDay Saints Hospital, Salt Lake City, Utah

\begin{abstract}
Object. The purpose of this study was to determine the effect of static and dynamic collimator optimization when using a micromultileaf collimator (mMLC) in dynamic-arc stereotactic radiosurgery (SRS) by evaluating the dose to healthy peritumoral tissue.

Methods. Thirty patients previously treated for intracranial lesions with the BrainLAB mMLC underwent retrospective replanning. Three collimator optimization strategies were compared for a simulated SRS treatment plan, as follows: Strategy 1, static collimation fixed at $90^{\circ}$ throughout arcs; Strategy 2, static collimator settings optimized for each arc; and Strategy 3, dynamic collimator settings optimized every $10^{\circ}$ throughout treatment arcs. Dose-volume histograms for a $0.7-\mathrm{cm}$ shell of healthy peritumoral tissue were quantitatively compared.

Collimator optimization schemes (Strategies 2 and 3) significantly decreased the volume of peritumoral tissue that is irradiated when compared with static collimation at $90^{\circ}$ (Strategy 1). The volume was reduced by $40.6 \%$ for Strategy $2(95 \%$ confidence interval $[\mathrm{CI}] \pm 11)$ and by $47.1 \%$ for Strategy $3(95 \% \mathrm{CI} \pm 8.1)$ at the $95 \%$ isodose; by $28.4 \%$ for Strategy 2 (95\% CI \pm 4.9$)$ and 39.1\% for Strategy $3(95 \%$ CI \pm 6$)$ at the 90\% isodose; and by 18.2\% for Strategy 2 $(95 \% \mathrm{CI} \pm 8.1)$ and $25.4 \%$ for Strategy $3(95 \% \mathrm{CI} \pm 7.1)$ at the $80 \%$ isodose. Serial collimator optimization throughout the treatment arcs (Strategy 3 ) reduced the mean volume of peritumoral tissue irradiated when compared with static collimator optimization (Strategy 2), by $16.1 \%(95 \% \mathrm{CI} \pm 1.5)$ at $95 \%$ isodose, by $11.7 \%(95 \% \mathrm{CI} \pm 1)$ at $90 \%$ isodose, and by $8.2 \%(95 \% \mathrm{CI} \pm 1.2)$ at $80 \%$ isodose regions. In specific cases, linear or polynomial functions were formulated to optimize collimator settings dynamically throughout treatment arcs.

Conclusions. Dynamic collimator optimization during arc-based SRS decreases the volume of healthy peritumoral tissue treated with high doses of radiation and appears to be an effective method of improving target conformality. This study is the first step toward determination of a smoothing function algorithm to allow for true dynamic collimation during SRS.
\end{abstract}

KEY WORDS • collimator optimization • stereotactic radiotherapy $\quad$ radiosurgery

The LINAC-based SRS and radiotherapy delivery systems enjoy widespread use in both academic and private radiation therapy centers. ${ }^{6,7,14,15}$ Multiple arcing beam techniques using circular collimators or mMLCs are most commonly used to deliver these highly conformal treatments. Previously reported data indicate that, except for spherical targets, the use of mMLCs improves both dose homogeneity within the target and sparing of healthy peritumoral tissue. ${ }^{1,3}$ The sparing of healthy tissues is of particular importance for intracranial lesions abutting critical, unaffected structures such as the optic nerves, chiasm, and brainstem. 1,3,4,6

Methods for further improvement of the target confor-

Abbreviations used in this paper: $\mathrm{CI}=$ confidence interval; $\mathrm{CT}=$ computerized tomography; $\mathrm{DVH}=$ dose-volume histogram; LINAC $=$ linear accelerator; mMLC $=$ micromultileaf collimator; $\mathrm{SRS}=$ stereotactic radiosurgery. mality and, therefore, sparing of healthy tissue by using mMLCs have been previously described. These include designing increasingly smaller leaf thicknesses $(5,4,3$, and $1.7 \mathrm{~mm}$ ); dynamic leaf motions during arcing (dynamic arcs); and optimization of the collimator settings to minimize the projected area of the target as seen by a beam'seye view. ${ }^{2-4,8-11}$ Kubo, et al..${ }^{9}$ reported a reduction in rectal wall and bladder dose for prostate radiotherapy plans by using a 3-mm mMLC compared with standard LINAC 10$\mathrm{mm}$ leaves. Nevertheless, DVHs of healthy tissues were only marginally improved for plans generated with $1.7-\mathrm{mm}$ leaves compared with the 3-mm mMLC. In recently published data, Monk, et al. ${ }^{12}$ suggest that for a wide range of target volumes $\left(0.8-46.7 \mathrm{~cm}^{3}\right)$, the difference in sparing of healthy tissue between 3- and 5-mm mMLCs may not be clinically significant.

In this paper, we evaluated the effect of dynamic collimator optimization during arc-based SRS for intracranial 


\section{M. Lee, G. A. Watson, and D. D. Leavitt}

malignancies. More specifically, we evaluated the effect of dynamic collimator optimization on healthy peritumoral tissues included within high-dose regions (between the 80 and $100 \%$ isodose lines). This study is the first step toward determination of a smoothing function algorithm to allow for true dynamic collimation during SRS.

\section{CLINICAL MATERIAL AND METHODS}

Thirty patients previously treated in our department with SRS underwent retrospective replanning in which three defined collimator optimization strategies were used. The median tumor volume was $8.2 \mathrm{~cm}^{3}$ (range $\left.0.1-36.7 \mathrm{~cm}^{3}\right)$. These analyses were made using the BrainLAB mMLC attached as an accessory to the LINAC unit (Clinac 2100 CD; Varian Oncology Systems, Palo Alto, CA). Initial planning CT data for patients were recorded with the use of a BrainLAB CT localizer frame and the data were digitally transported to the BrainSCAN treatment planning software. Target volumes for each patient were outlined on axial fused CT/magnetic resonance images, and beam'seye views were used to define micromultileaf beam shapes.

Treatment planning was performed for each patient with five dynamic SRS arcs around a single isocenter. The DVH calculations were performed with a $0.5-\mathrm{mm}$ grid size by using BrainSCAN 5.0 SRS planning software. Arcs were converted to multiple static fields at $10^{\circ}$ intervals throughout the rotational path to simulate a dynamic collimation method. The DVHs for a $0.7-\mathrm{cm}$-thick shell of healthy peritumoral tissue were quantitatively compared. Isodose distributions and DVHs were plotted for comparison between plans with optimized and nonoptimized (fixed at $90^{\circ}$ ) collimator settings. Because the volume of healthy peritumoral tissue that was irradiated was used as a criterion to assess each optimization strategy, we have chosen in this study to evaluate volumes contained within high-dose regions (defined specifically as $80-100 \%$ isodose lines).

Three collimator optimization schemes (Strategies 1-3) were compared for a simulated SRS treatment plan as follows: Strategy 1, static collimation with the collimator fixed at $90^{\circ}$ throughout each arc; Strategy 2, static collimator settings fixed at the computed optimized angle for each SRS arc; and Strategy 3, dynamic collimator optimization throughout each arc rotation (theoretical plan with fixed beams optimized at each $10^{\circ}$ increment throughout treatment arcs). Strategy 1 was chosen for comparison because it uses the BrainSCAN 5.0 SRS software preset with the collimator fixed at $90^{\circ}$ throughout the treatment arc (static unoptimized fields). The Varian LINAC unit is configured using Varian standard notation. In this configuration, a collimator angle of $90^{\circ}$ corresponds to leaf motion coincident with the direction of gantry rotation. Strategy 2 for collimator optimization determines an optimal fixed collimator setting for each arc with computational software/algorithms that determine the collimator angle at which the smallest composite beam's-eye view area is found. Strategy 3 for collimator optimization allows for analysis of a simulated, dynamically collimated arc (theoretical plan). In each patient in whom the third strategy was used, static, conformal fields at $10^{\circ}$ increments were positioned throughout the arc path. The optimal collimator angle for each static field was then calculated by minimization of the area of the beam'seye view for each increment.
Parameters used to compare the three collimator optimization strategies were the percentage of healthy peritumoral tissue encompassed by the 80 to $100 \%$ isodose lines (defined as high-dose regions). The DVHs for the peritumoral tissue shell were obtained for each retroplanned target with each of the collimator optimization strategies. The statistical significance of each comparison was evaluated using the paired two-tailed Student t-test.

After determination of serial optimized collimator angles across each gantry arc at $10^{\circ}$ increments (Strategy 3 ), linear and/or polynomial functions were calculated to fit the resulting data points. These resulting functions were calculated to enable theoretical simulation of how the collimator could dynamically change angles throughout a specific treatment arc. To determine the maximal rotational velocity allowable for the collimator and gantry with the Varian LINAC unit, serial measurements were obtained.

\section{RESULTS}

Figure 1A and $\mathrm{B}$ reveal how the three collimator optimization strategies affect the DVHs of the healthy peritumoral tissue and the target tissue differently. Figure 1A illustrates a representative example of varied percentages of healthy peritumoral tissue volume $(0.7-\mathrm{cm}$ shell surrounding the target) that receive a high dose of radiation (between the 80 and 100\% isodose lines) for each of the collimator optimization strategies. These differences are subsequently contrasted with Fig. 1B, which reveals similar overall DVHs for the target among each of the three collimator optimization strategies.

Figure 2 illustrates the resulting difference in the mean percentage of peritumoral tissue sparing after both static optimization (Strategy 2) and dynamic collimator optimization (Strategy 3) compared with a fixed $90^{\circ}$ collimator setting (Strategy 1). Collimator optimizations (Strategies 2 and 3) significantly decrease the volume of peritumoral tissue included within the high-dose regions (defined as the $80-100 \%$ isodose line) compared with static collimation at $90^{\circ}$; by $40.6 \%(95 \% \mathrm{CI} \pm 11)$ and $47.1 \%(95 \% \mathrm{CI} \pm 8.1)$ at $95 \%$ isodose, $28.4 \%(95 \% \mathrm{CI} \pm 4.9)$ and $39.1 \%(95 \% \mathrm{CI}$ $\pm 6)$ at $90 \%$ isodose, and $18.2 \%(95 \% \mathrm{CI} \pm 8.1)$ and $25.4 \%(95 \% \mathrm{CI} \pm 7.1)$ at $80 \%$ isodose, respectively.

Simulated dynamic collimator optimization throughout the treatment arcs (Strategy 3) reduced the mean volume of peritumoral tissue irradiated when compared with static collimator optimization (Strategy 2); by $16.1 \%$ (95\% CI \pm $1.5)$ at $95 \%$ isodose, $11.7 \%(95 \% \mathrm{CI} \pm 1)$ for $90 \%$ isodose, and $8.2 \%$ (95\% CI \pm 1.2 ) for $80 \%$ isodose regions (Fig. 3). This resulted in a small but significant amount of peritumoral tissue sparing. A scatterplot was constructed to evaluate the relationship of tumor volume to the percentage of healthy peritumoral tissue spared (Fig. 4A). An additional graph was constructed to illustrate the relationship between target shape/roundness and percentage of peritumoral tissue spared (Fig. 4B). Although the resulting correlation is poor between tumor volume and peritumoral tissue sparing, Fig. 4A reveals a general trend toward increased sparing of healthy peritumoral tissue with increased tumor volumes. We did not find a significant correlation between target roundness and sparing of peritumoral tissue. This nonsignificant correlation is mainly due to the fact that 


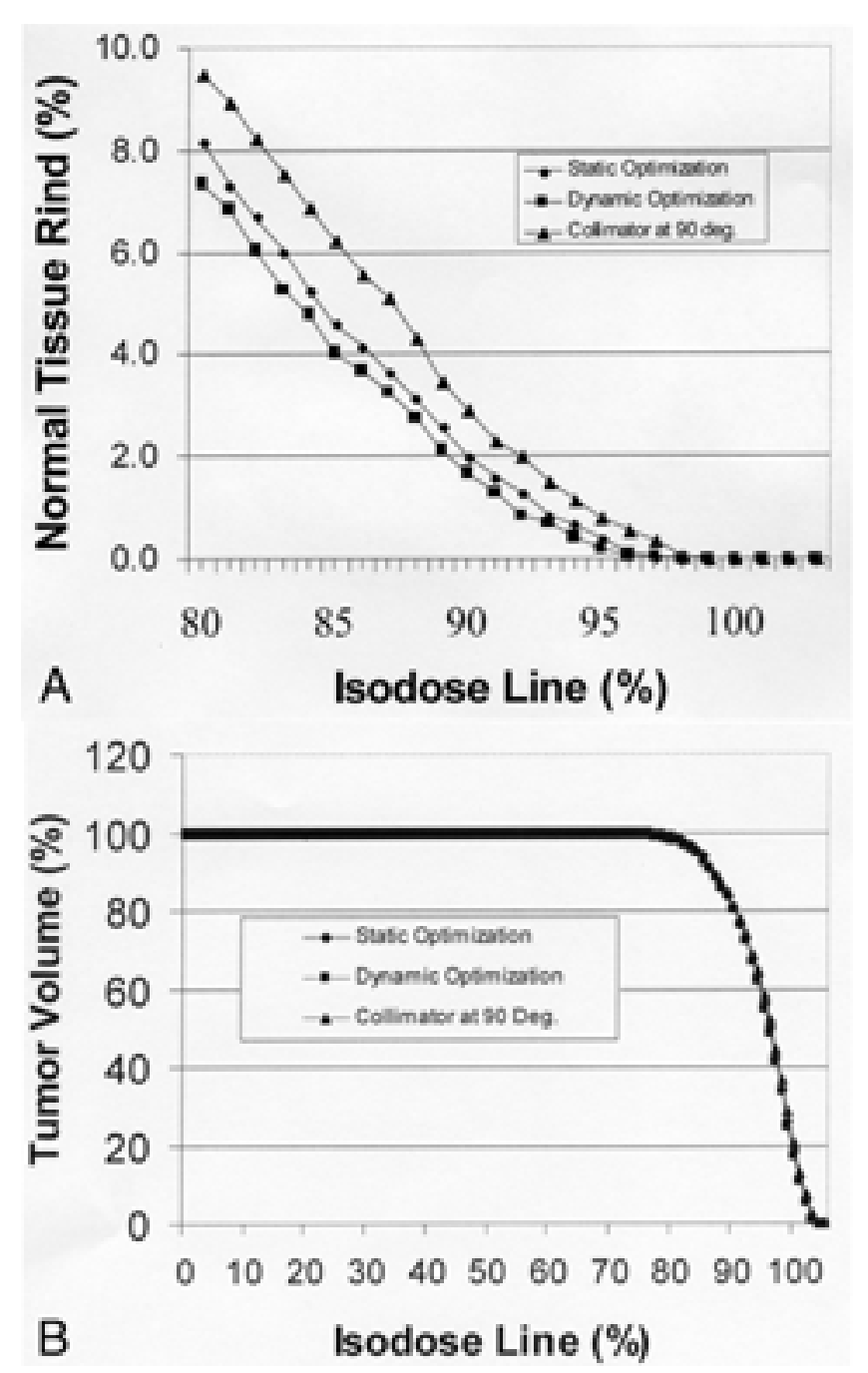

Fig. 1. Graphs showing a representative example of DVHs for an irregularly shaped tumor. A: Percentage of normal (healthy) tissue rind within the high-dose region ( $80-100 \%$ isodose lines). B: Percentage of tumor volume (0-100\% isodose lines).

multiple concave surfaces were found on the target structure. In general, it is believed that the three-dimensional complexity of the target volume would better correlate with decreased sparing of healthy tissue.

In specific cases, linear or polynomial functions were formulated to simulate how a dynamically optimized collimator would change angles throughout specific treatment arcs. Figure 5A illustrates an example of an arc in which the change in gantry angle compared with collimator angle had a linear fit $[y=-3.2143(x)+163.57]$. After the linear fit was defined, the resulting amount of healthy peritumoral tissue spared was significantly improved $(\mathrm{p}=0.022)$. Figure 5B is an example of a second-degree polynomial fit $\left[y=-0.8683\left(x^{2}\right)+11.192(x)+101.88\right]$, which represents change in collimator angle compared with change in gantry angle across an arc. Although not all tumor shapes allow feasible polynomial functions to be fit, in specific cases healthy peritumoral tissue can be spared a significant radiation dose by this theoretical method. Maximal collimator

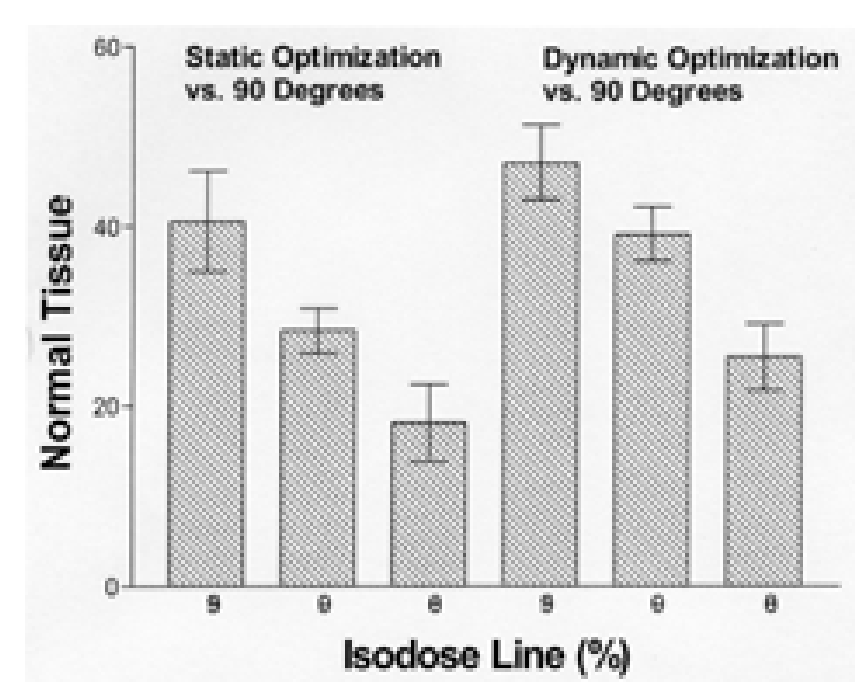

Fig. 2. Bar graph showing that the percentage of healthy peritumoral tissue included within the defined high-dose regions (80-100\% isodose regions) is dependent on the collimator optimization strategy used.

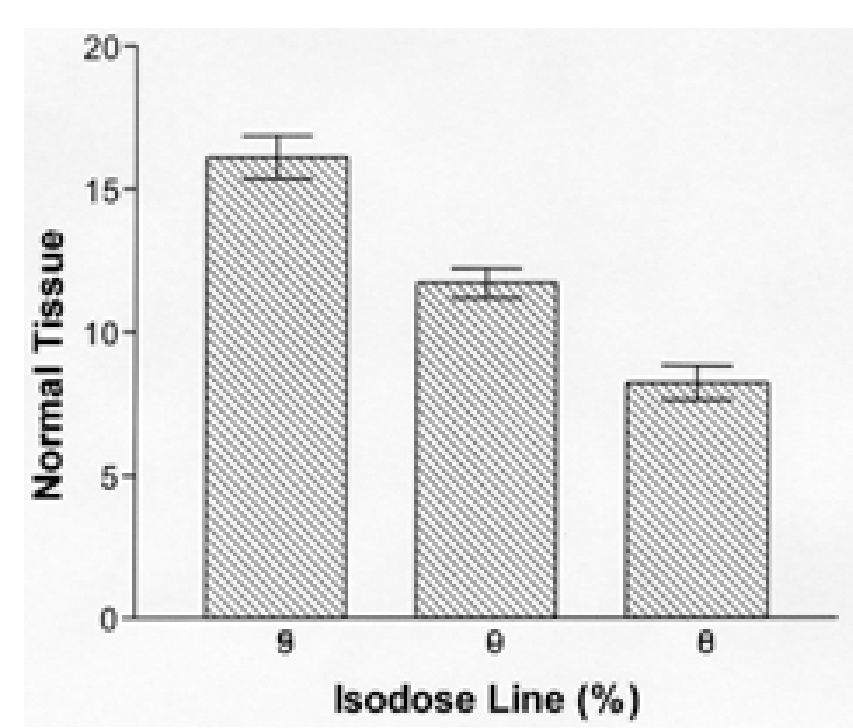

Fig. 3. Bar graph showing improved sparing of healthy peritumoral tissue included within high-dose regions (80-95\% isodose lines) with dynamic collimator optimization (Strategy 3) when compared with static collimator optimization (Strategy 2).

rotational velocity for the Varian LINAC unit was measured at $3.6^{\circ}$ per second, and maximal gantry rotational velocity was measured at $7.2^{\circ}$ per second.

\section{DISCUSSION}

Dynamic collimator optimization during arc-based SRS decreases the volume of healthy peritumoral tissue treated with high doses when compared with fixed collimator optimization strategies. Although this dosimetric study is only computational and theoretical in nature, dynamic collima- 


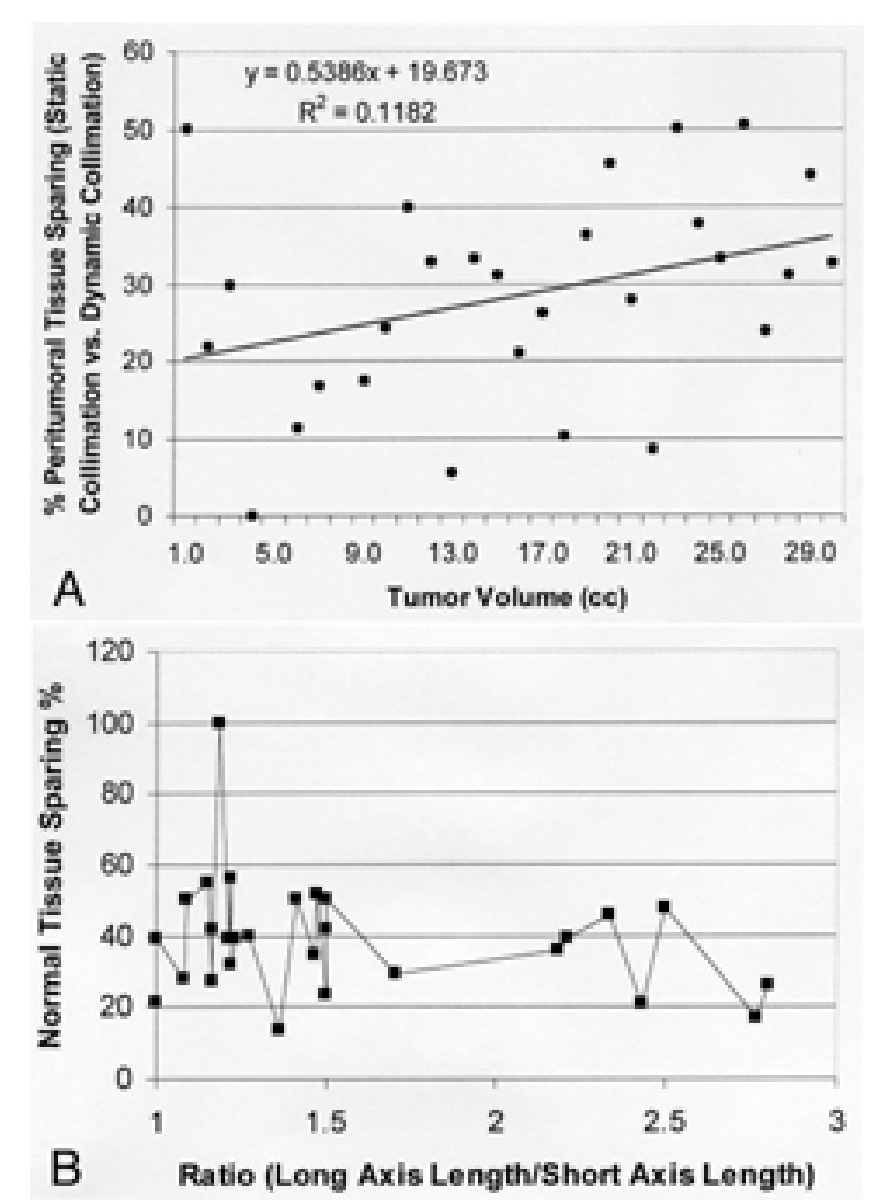

Fig. 4. A: Scatterplot showing the effect of relative tumor volume on the percentage of healthy peritumoral tissue that was spared the dose within the high-dose regions (80-100\% isodose lines). B: Graph showing the relationship between the roundness function of target shape and healthy peritumoral tissue that was spared within the high-dose regions (80-100\% isodose lines).

tor optimization appears to be an effective method of improving target conformality. Whereas the principal goal of SRS is to provide a method of focal irradiation of target tissue to higher doses without increasing complications in healthy tissue, dynamic collimator optimization may provide a feasible means by which this may be accomplished. This follows logically from the assumption that the toxicity of such techniques is determined by the dose and volume of healthy tissue irradiated.

Actual measurements comparing resulting dose distributions for the various collimator optimization strategies have not been performed because this is purely a theoretical planning study. Many questions remain as to the feasibility of dynamic collimator optimization along an arc's rotation. Investigations into the mechanical stability of the LINAC across a treatment arc as well as determination of possible setup errors will need to be completed. The variability in the reproducibility of patient setup with pin fixation or other head immobilization devices has been asserted to be much less than that encountered with conventional radiotherapy techniques. ${ }^{1,3}$

As revealed in these experiments, the maximal rotational
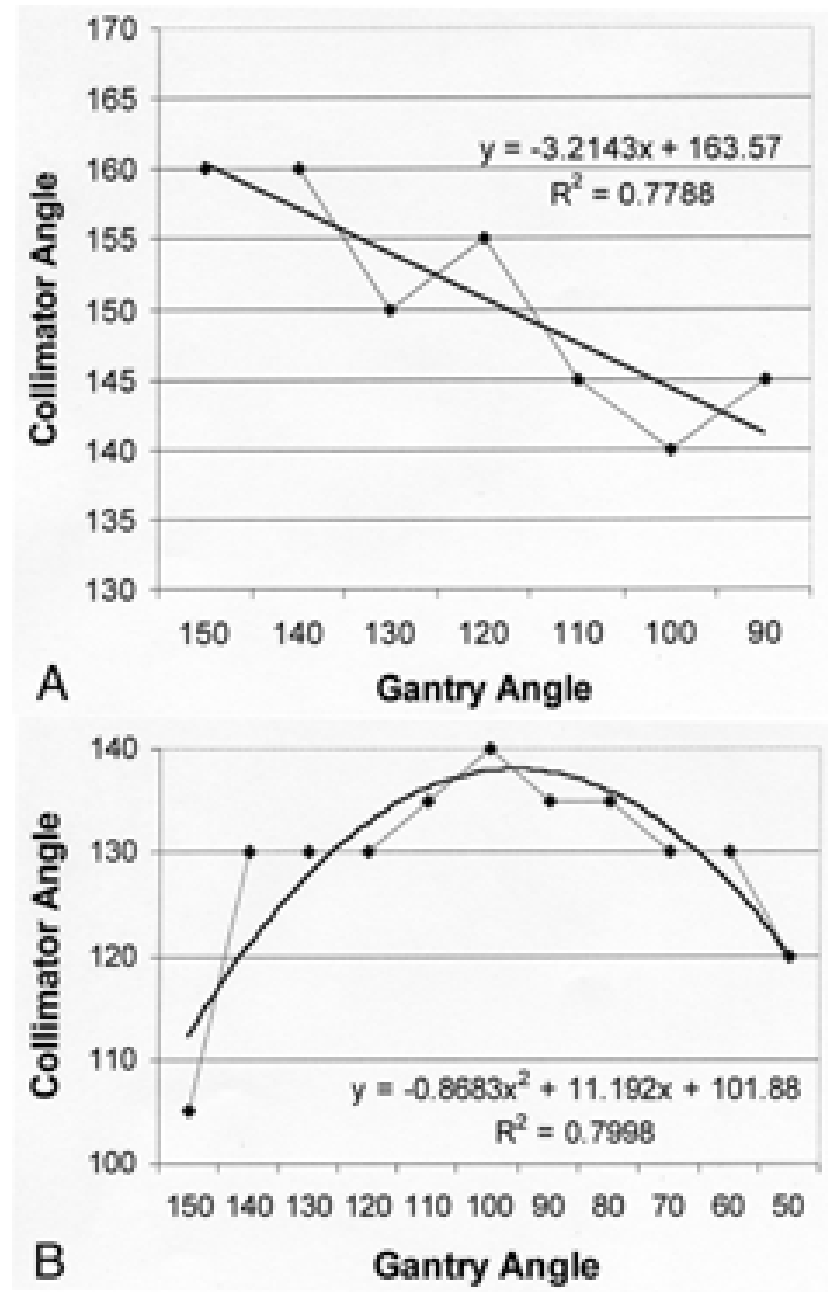

Fig. 5. A: Graph showing theoretical dynamic collimator optimization function for a radiosurgery arc in which the change in gantry angle compared with collimator angle had a linear fit. B: Graph showing theoretical dynamic collimator optimization function for a radiosurgery arc in which the change in gantry angle compared with collimator angle had a second-degree polynomial fit.

rate of the collimator for the Varian LINAC unit was measured at $3.6^{\circ}$ per second, and maximal gantry rotational velocity was measured at $7.2^{\circ}$ per second. The ability to alter the collimator angle smoothly may be limited by a particular LINAC's maximal controlled collimator rotational velocity. We found that in such cases as illustrated in Fig. 5, the desired collimator rotational speeds would be feasible, although this would not be the case for all tumor shapes and/or locations. For treatment with constant radiation output, the speed of gantry rotation also sets the minimum dose delivered per degree of arc (typically 2-10 monitor units/ degree). Theoretically, increasing the number of arcs for a particular treatment plan also significantly reduces the integral dose received by healthy surrounding tissue while providing more uniform target volume coverage. Nevertheless, departmental or patient time constraints can be an issue for each additional arc rotation included with a plan. $.3,4,6,14,15$ The mean gantry rotational velocity would also be slower for a 
single-fraction radiosurgery case when compared with fractionated SRS, thereby indicating that a greater number of single-fraction radiosurgery treatments could use dynamic collimator optimization.

Although dosimetric differences do exist within the adjacent peritumoral target tissue between variations in collimator optimization strategies, the clinical importance of these small differences is unclear. It is accepted that the radiotolerance and resulting toxicity in each organ at risk is dependent on the volume that is contained within regions receiving high doses. Depending on the clinical and dosimetric goals in a specific case, small decreases in the amount of healthy peritumoral tissue treated with high doses of radiation may be deemed advantageous by clinicians. Currently, DVHs give clinicians the best volumetric comparison between treatment plans. It is apparent, however, that the position of sensitive eloquent brain structures relative to the target volume may create a situation in which sparing radiation doses to critical healthy tissues is clinically more important than obtaining the optimal target DVH. The volume of healthy peritumoral tissue irradiated can also be used as a criterion to assess toxicity, and there is a choice as to which isodose should be compared. In this particular study, we decided to assess volumes of healthy tissue included within high-isodose regions $(80-100 \%$ isodose lines). It is generally accepted that clinically measurable late toxicity would be incurred within this region. ${ }^{2,5,7,12,13}$ Nevertheless, the precise relationship between dose, volume, and toxicity has not been well described. We found in our analysis that features such as target size and roundness did not have statistically significant correlates with peritumoral tissue sparing (Fig. 4).

Although dose conformation could be improved for each case by our theoretical model, the ability to perform firstthrough fourth-degree polynomial fits for specific arcs is limited by tumor shape and location within the cranial cavity. As illustrated in Fig. 5, first- and third-degree polynomial fits are all that is needed in specific cases for further dose optimization. The particular polynomial function that best fits a designated arc is dependent on the target shape and specific positions of the collimator across the rotational arc. Nevertheless, for other particular cases, first- through fourth-degree polynomial fits were unable to provide an appreciable benefit. Further research will also be needed to facilitate automated determination of smoothing function algorithms and their individual feasibility.

We have presented an analysis of the comparison of treatment plans for intracranial lesions treated with SRS with three different collimator optimization strategies. Dynamic collimator optimization during arc-based SRS was found to decrease the percentage of healthy peritumoral tissue treated in high-dose regions, and hence target conformality was improved. These findings have immediate application in the ongoing research to develop a smoothing function algorithm to allow for true dynamic collimation during dynamic-arc SRS in which an mMLC is used. In addition, future research will be aimed at performing a cost/function analysis with the following factors: conformality factor, peritumoral tissue sparing factor, object at risk proximity factor, treatment calculation time factor, and treatment time factor. The goal is to provide an objective determination of the benefit from increasingly complex and time-intensive radiation treatment planning and delivery.

\section{Disclaimer}

The authors have no financial interest in any of the equipment or processes mentioned in this paper.

\section{References}

1. Bourland JD, McCollough KP: Static field conformal stereotactic radiosurgery: physical techniques. Int J Radiat Oncol Biol Phys 28:471-479, 1994

2. Boyer AL: Radiation therapy beam modulation techniques. Cancer Treat Res 93:35-48, 1998

3. Chierego G, Francescon P, Colombo F, et al: From radiotherapy to stereotactic radiosurgery: physical and dosimetrical considerations. Radiother Oncol 29:214-218, 1993

4. Das IJ, Downes MB, Corn BW, et al: Characteristics of a dedicated linear accelerator-based stereotactic radiosurgery-radiotherapy unit. Radiother Oncol 38:61-68, 1996

5. Fiveash JB, Murshed H, Duan J, et al: Effect of multileaf collimator leaf width on physical dose distributions in the treatment of CNS and head and neck neoplasms with intensity modulated radiation therapy. Med Phys 29:1116-1119, 2002

6. Friedman WA, Foote KD: Linear accelerator radiosurgery in the management of brain tumors. Ann Med 32:64-80, 2000

7. Heron DE, Godette KD, Wynn RA, et al: Radiation medicine innovations for the new millenium. J Natl Med Assoc 95: 55-63, 2003

8. Kubo HD, Pappas CT, Wilder RB: A comparison of arc-based and static mini-multileaf collimator-based radiosurgery treatment plans. Radiother Oncol 45:89-93, 1997

9. Kubo HD, Wilder RB, Pappas CT: Impact of collimator leaf width on stereotactic radiosurgery and 3D conformal radiotherapy treatment plans. Int J Radiat Oncol Biol Phys 44: 937-945, 1999

10. Leavitt DD: Beam shaping for SRT/SRS. Med Dosim 23: 229-236, 1998

11. Leavitt DD, Gibbs FA Jr, Heilbrun MP, et al: Dynamic field shaping to optimize stereotactic radiosurgery. Int J Radiat Oncol Biol Phys 21:1247-1255, 1991

12. Monk JE, Perks JR, Doughty D, et al: Comparison of a micromultileaf collimator with a 5-mm-leaf-width collimator for intracranial stereotactic radiotherapy. Int J Radiat Oncol Biol Phys 57:1443-1449, 2003

13. Nedzi LA, Kooy HM, Alexander E III, et al: Dynamic field shaping for stereotactic radiosurgery: a modeling study. Int J Radiat Oncol Biol Phys 25:859-869, 1993

14. Regine WF: The radiation oncologist's perspective on stereotactic radiosurgery. Technol Cancer Res Treat 1:43-49, 2002

15. Tome WA, Mehta MP, Meeks SL, et al: Fractionated stereotactic radiotherapy: a short review. Technol Cancer Res Treat 1:153-172, 2002

\section{Manuscript received December 17, 2004.}

Accepted in final form May 31, 2005.

Presented at the LINAC Radiosurgery Scientific Symposium, December 4-8, 2002, Lake Buena Vista, Florida.

Address reprint requests to: Gordon A. Watson, M.D., Ph.D., Department of Radiation Oncology, LDS Hospital, 8th Avenue and C Street, Salt Lake City, Utah 84143. email: gawatson@ihc.com. 